\title{
A phase-compensated distorted wave Born approximation representation of the bistatic scattering by weakly scattering objects: Application to zooplankton
}

\author{
Dezhang $\mathrm{Chu}^{\mathrm{a})}$ \\ Department of Applied Ocean Physics and Engineering, Woods Hole Oceanographic Institution, \\ Woods Hole, Massachusetts 02543 \\ Zhen Ye \\ Department of Physics, National Central University, Chung-li, Taiwan, Republic of China
}

(Received 13 November 1998; revised 26 May 1999; accepted 8 June 1999)

\begin{abstract}
The distorted wave Born approximation (DWBA) method has been successfully used in modeling the acoustic backscattering by weakly scattering zooplankton [Stanton et al., J. Acoust. Soc. Am. 94, 3463-3472 (1993), Wiebe et al., IEEE J. Ocean. Eng. 22(3), 445-464 (1997)]. However, the previously developed DWBA model ignores the imaginary part of the scattering amplitude and thus results in a zero-extinction cross section. As a consequence, the model fails to predict the scattering-induced attenuation which could be important under certain circumstances. In this paper, a phase-compensated DWBA-based approximation is presented. The improved method allows us to compute not only the scattering strength but also the acoustic attenuation. The new scattering model is validated by comparing with the existing exact solution for certain representative finite objects. The results from this study can be applied to bioacoustic applications where the attenuation due to scattering and/or multiple scattering by zooplankton is relevant, and where this information might be used to infer the acoustic properties of live animals. (c) 1999 Acoustical Society of America. [S0001-4966(99)00410-5]
\end{abstract}

PACS numbers: 43.30.Ft, 43.30.Xm, 43.30.Gr, 43.20.Fn [DLB]

\section{INTRODUCTION}

Mathematical modeling of the acoustical scattering by individual zooplankton has evolved from the simplest sphere model $^{1}$ to the recent distorted wave Born approximation (DWBA), ${ }^{2,3}$ which is capable of describing the acoustic scattering by arbitrarily shaped inhomogeneous scatterers. The DWBA model has been successfully applied to both laboratory and shipboard experimental data, ${ }^{3,4}$ as well as field data. ${ }^{5}$ However, when the scattering-induced attenuation is noticeable and cannot be ignored, such as in the case of scattering by densely aggregated zooplankton, the conventional DWBA fails to describe the scattering characteristics. To illustrate this, consider a plane wave propagating through a random medium as shown in Fig. 1. The attenuation through the medium can be described in terms of the extinction cross section $\sigma_{e}(\mathbf{p}):^{6}$

$$
I_{s}(z)=I_{0} e^{-z} \int_{\mathbf{p}} \rho_{\mathbf{p}} \sigma_{e}(\mathbf{p}) d_{\mathbf{p}},
$$

where $I_{0}$ is the original acoustic intensity at $z=0$ while $I_{s}$ is the observed intensity at $z \cdot \rho(\mathbf{p})$ is the number of scatterers in a unit volume with parameter $\mathbf{p}$ referring to the properties of the aggregated scatterers. According to the forwardscattering theorem, ${ }^{7}$ the extinction cross section $\sigma_{e}(\mathbf{p})$ can be expressed in terms of the imaginary part of the scattering amplitude in the forward direction as

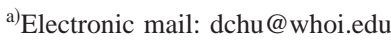

$$
\sigma_{e}=\operatorname{Im}\left(\frac{4 \pi f_{\mathbf{p}}(\hat{0})}{k}\right),
$$

where $k$ is the acoustic wave number of the incident wave in the surrounding medium, and $f_{\mathbf{p}}(\hat{0})$ is the forward-scattering amplitude of the individual scatterer with parameter $\mathbf{p}$. Equation (2) indicates that the extinction cross section $\sigma_{e}$ is proportional to the imaginary part of the scattering amplitude in the forward direction. However, because of its inherent deficiency, the DWBA fails to predict the imaginary part of the scattering amplitude even for some regularly shaped targets such as spheres. To overcome this shortcoming, a modified DWBA model is proposed in this paper, which adds a heuristic phase compensation term. The phase-compensated DWBA solution is able to estimate the extinction cross section using the aforementioned forward-scattering theorem. The paper is organized in the following way. Section I briefly reviews the Born and DWBA models, while Sec. II provides the theoretical development of the phasecompensated DWBA model. In Sec. III, the results derived in Sec. II are extended to a more general case and then applied to zooplankton scattering. Simulation results are presented and discussed. Finally, conclusions are drawn in Sec. VI.

\section{BORN APPROXIMATION (BA) AND DISTORTED WAVE BORN APPROXIMATION (DWBA)}

Before introducing the Born approximation (BA) and distorted wave Born approximation (DWBA), consider a unit plane wave, $e^{i \mathbf{k} \cdot \mathbf{r}}$, impinging on a volumetric foreign body 


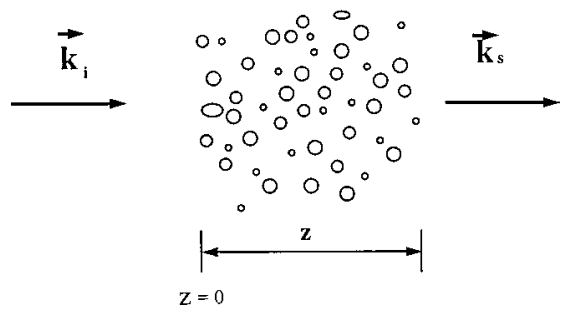

FIG. 1. Scattering by a cloud of randomly distributed fluid scatterers due to a plane incident wave.

(scatterer), $V$, whose acoustic properties are different from those of the surrounding medium. The integral equation representation of the scattering by such a volume is given by ${ }^{6}$

$$
\begin{aligned}
p_{\text {scat }}= & \int_{V}\left[k^{2} \gamma_{k} P_{\text {int }}\left(\mathbf{r}_{0}\right) G\left(r \mid \mathbf{r}_{0}\right)\right. \\
& \left.+\gamma_{\rho} \nabla P_{\text {int }}\left(\mathbf{r}_{0}\right) \cdot \nabla G\left(\mathbf{r} \mid \mathbf{r}_{0}\right)\right] d \mathbf{v}_{0},
\end{aligned}
$$

where $P_{\text {int }}\left(\mathbf{r}_{0}\right)$ and $G\left(\mathbf{r} \mid \mathbf{r}_{0}\right)$ are internal field and free-space Green's function, respectively, with $\mathbf{r}_{0}$ representing the position vector within the volume $V . \gamma_{k}$ and $\gamma_{\rho}$ are defined as

$$
\gamma_{k}=\frac{\kappa_{1}-\kappa}{\kappa_{1}}=\frac{1-g h^{2}}{g h^{2}}, \quad \gamma_{\rho}=\frac{\rho_{1}-\rho}{\rho_{1}}=\frac{g-1}{g},
$$

where $\kappa=1 / \rho c^{2}$ is the compressibility and the subscript " 1 ", refers to the inhomogeneous volume, $\rho$ and $c$ are respectively density and sound speed in the surrounding medium. $g$ $=\rho_{1} / \rho$ and $h=c_{1} / c$ are density and sound-speed contrasts of the inhomogeneous volume to the surrounding medium, respectively. Since the internal field $P_{\text {int }}$ is unknown, the exact scattering field has to be obtained by solving the integral equation. In reality, most scatterers have complex shapes with inhomogeneous acoustic properties within their bodies; it is nearly impossible to obtain exact solutions for scattering by such objects. Approximation schemes have to be resorted to in order to estimate the acoustic scattering response. In particular, if the internal field in Eq. (3) can be represented by an analytical function, the integral equation reduces to an integral that can be evaluated directly.

\section{A. Born approximation}

For weakly scattering $g \approx 1$ and $h \approx 1$, which are the cases for most zooplankton applications, one of the commonly used approximations is the well-known Born approximation (BA). ${ }^{8}$ In the BA, the internal field $P_{\text {int }}\left(\mathbf{r}_{0}\right)$ in Eq. (3) is replaced with the incident field, $e^{i \mathbf{k}_{i} \cdot \mathbf{r}_{0}}$, since it is very close to the incident field with the absence of scatterers. To evaluate the performance of the BA, we compare it with the exact solutions for backscattering by a fluid sphere. The scattering geometry is shown in Fig. 2, where $\theta_{s}=180^{\circ}$ for backscattering. Substituting approximate internal field $P_{\text {int }}\left(\mathbf{r}_{0}\right)=e^{i \mathbf{k}_{i} \cdot \mathbf{r}_{0}}$ into Eq. (3) and using the free-space Green's function

$$
\begin{aligned}
G\left(\mathbf{r} \mid \mathbf{r}_{0}\right) & =e^{i k\left|\mathbf{r}-\mathbf{r}_{0}\right| / 4 \pi\left|\mathbf{r}-\mathbf{r}_{0}\right|} \\
& \approx e^{i k r} e^{-i \mathbf{k}_{s} \cdot \mathbf{r}_{0} / 4 \pi r,} \quad \text { for }|\mathbf{r}| \gg\left|\mathbf{r}_{0}\right|,
\end{aligned}
$$

where $\left|\mathbf{k}_{i}\right|=\left|\mathbf{k}_{s}\right|=k$, we obtain

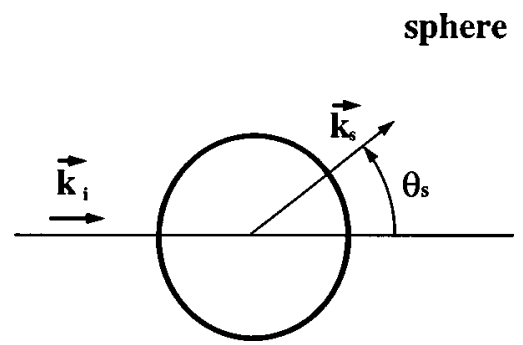

FIG. 2. Geometry of the bistatic scattering by a sphere. $\theta_{s}=0^{\circ}$ for forward scattering and $\theta_{s}=180^{\circ}$ for backscattering.

$$
\begin{aligned}
p_{\text {scat }}^{\mathrm{BA}} & =\frac{e^{i k r}}{r} \frac{k^{2}}{4 \pi} \int_{V}\left(\gamma_{k}+\hat{k}_{s} \cdot \hat{k}_{i} \gamma_{\rho}\right) e^{i\left(\mathbf{k}_{i}-\mathbf{k}_{s}\right) \cdot \mathbf{r}_{0}} d \mathbf{v}_{0} \\
& =\frac{e^{i k r}}{r} f_{\text {scat }}^{\mathrm{BA}}
\end{aligned}
$$

where the symbol " ", , stands for the unit vector, $f_{\text {scat }}^{\mathrm{BA}}$ is the scattering amplitude based on the BA and is found to be [Appendix, (A22)]

$$
f_{\text {scat }}^{\mathrm{BA}}\left(\theta_{s}\right)=k^{2} a^{3}\left(\gamma_{k}+\gamma_{\rho} \cos \theta_{s}\right) \frac{j_{1}\left(2 k a \sin \left(\theta_{s} / 2\right)\right)}{2 k a \sin \left(\theta_{s} / 2\right)},
$$

where $j_{1}(x)$ is the spherical Bessel function of order 1 and the scattering angle $\theta_{s}$ is the angle between the incident and scattering directions depicted in Fig. 2. The comparison for the backscattering $\left(\theta_{s}=180^{\circ}\right)$ between the exact partial wave (modal series) solution ${ }^{9}$ and the BA with different density and sound-speed contrasts $g$ and $h$ is given in Fig. 3. To make the results more representative, we have plotted the scattering form function $f_{\infty}$ (a dimensionless function) instead of the scattering amplitude. Since for a finite object the form function and the scattering amplitude are different only by a scaling constant, $a / 2$ for a sphere, the term "scattering amplitude" will be used for discussing scattering while the form function will be used for plotting. Three observations can be found by inspecting Fig. 3: (1) for $k a \ll 1$, BA provides very good fits in all cases. This is because when the acoustic wavelength is much longer than the object dimension, the internal field is essentially the same as that when the scatterer is absent; (2) the phase difference increases as sound-speed contrast $h$ and $k a$ increase. This is due to the fact that the phase difference is directly related to the travel time difference determined by the sound-speed contrast and has a larger impact as $k a$ increases; (3) the amplitude difference increases with $g$ and $h$ but maintains a regular oscillatory pattern over the entire $k a$ range. This phenomenon is expected since larger $g$ and $h$ imply stronger scattering, hence, the weak scattering assumption, on which the BA is based, may be violated.

\section{B. Distorted wave born approximation}

Apparently, the BA cannot provide satisfactory results even for a moderate $k a$. It can be shown that the amplitude mismatch is due to the exclusion of higher-order internal waves (multiple bounces within the scatterer). Since the characteristics of the higher-order scattering strongly depend on the shape and orientation of the scatterer, as well as the 

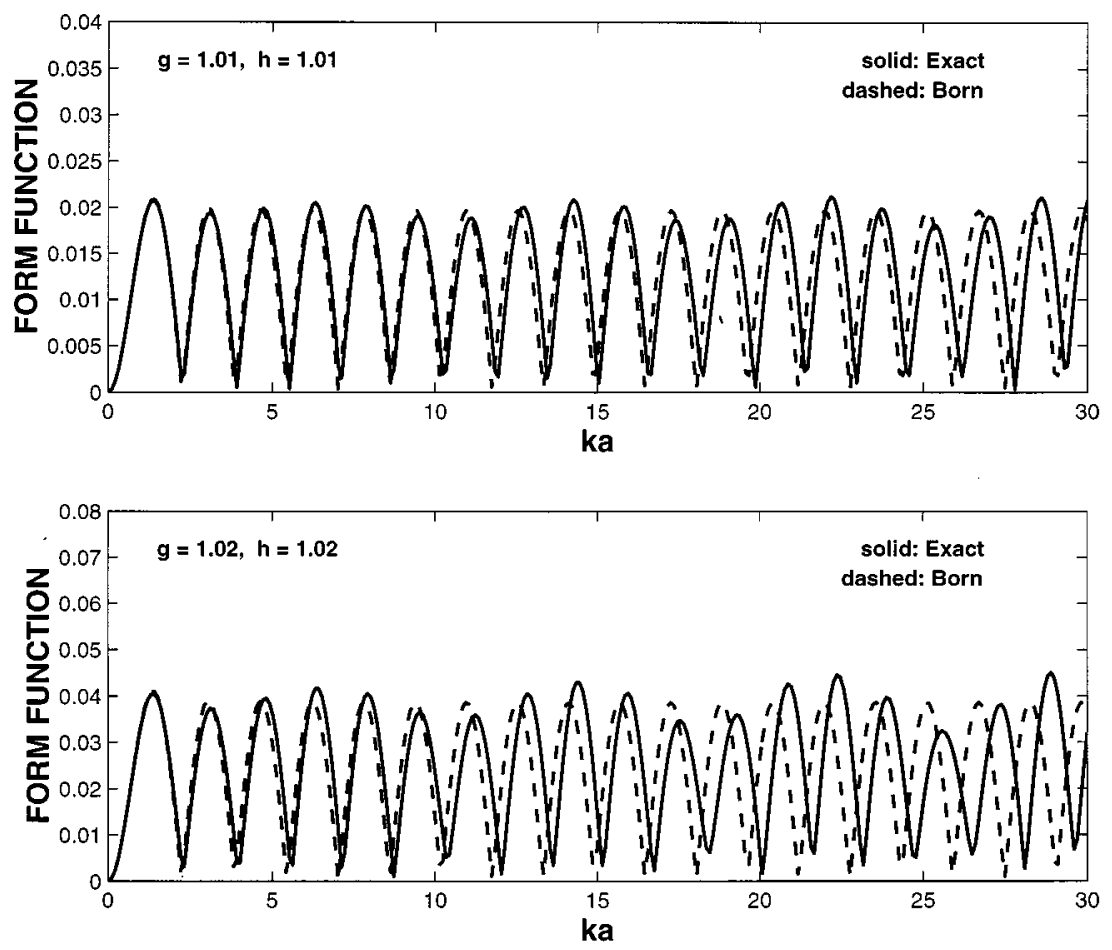

FIG. 3. Comparison of the backscattering form function $\left(\theta_{s}=180^{\circ}\right)$ of a fluid sphere between the exact solution and the Born approximation (BA) with different combinations of the density and sound-speed contrasts $(g$ and $h$ ). The solid lines are computed from the exact solution (Ref. 9) and the dashed lines are computed from Eq. (6).

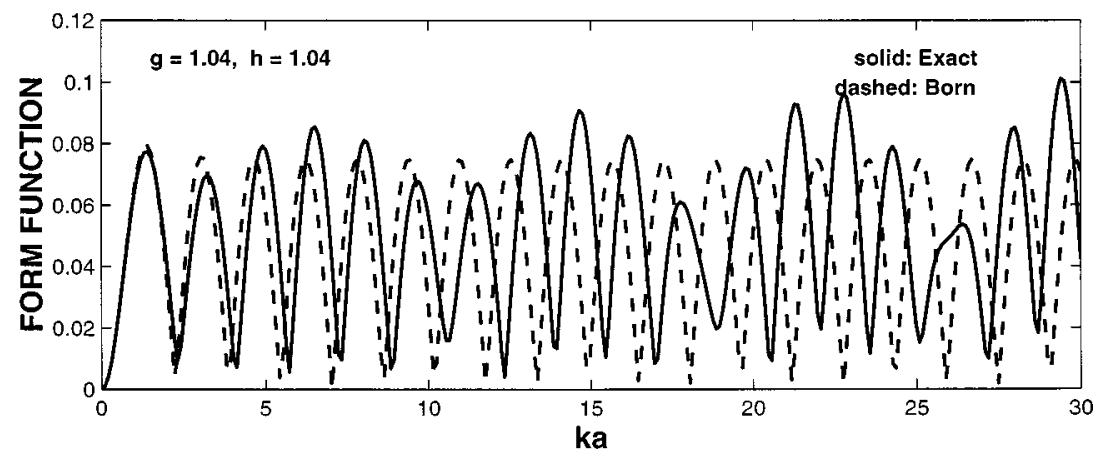

scattering geometry, it is very difficult to account for this amplitude mismatch. The phase mismatch, however, is essentially due to the sound-speed contrast and the scatterer's dimension in the direction of the incident wave, and is relatively easy to characterize. The distorted wave Born approximation (DWBA) is introduced to compensate such a phase mismatch. Mathematically, the DWBA replaces the internal field $P_{\text {int }}\left(\mathbf{k}_{0}\right)$ with the same function form as in the BA, but further replaces the wave vector $\mathbf{k}_{i}$ in the phase by $\mathbf{k}_{1}$, representing a distorted wave. In addition, a corresponding modification of the free-space Green's function is included. For such a distorted Green's function, the wave number in its exponential within the inhomogeneous volume is $\left|\mathbf{k}_{s}\right|=k_{1}$ $=k / h$. With these modifications, the scattering amplitude using the DWBA can be expressed as

$$
\begin{aligned}
f_{\text {scat }}^{\text {DWBA }}\left(\theta_{0}\right)= & \frac{k_{1}^{2}}{4 \pi} \int_{V}\left(h^{2} \gamma_{k}+\hat{k}_{i} \cdot \hat{k}_{s} \gamma_{\rho}\right) e^{i\left(\mathbf{k}_{i}-\mathbf{k}_{s}\right) \cdot \mathbf{r}_{0}} d \mathbf{v} \\
= & k_{1}^{2} a^{3}\left(h^{2} \gamma_{k}+\gamma_{\rho} \cos \theta_{s}\right) \\
& \times\left[\frac{j_{1}\left(2 k_{1} a \sin \left(\theta_{s} / 2\right)\right)}{2 k_{1} a \sin \left(\theta_{s} / 2\right)}\right] .
\end{aligned}
$$

The only major difference between Eq. (6) and Eq. (7) is that the wave numbers $k^{\prime} \mathrm{s}$ in the square-bracketed term are replaced by $k_{1}^{\prime} \mathrm{s}$. Although $\left|k_{1}\right| \approx k$ for weakly scattering, a slight change from $k$ to $k_{1}$ in the argument of the spherical Bessel function gives rise to a significant difference in the frequency response of the scattering, especially for large $k a$. Figure 4 shows the comparison between the exact solution and the DWBA for backscattering $\left(\theta_{s}=180^{\circ}\right)$. In Fig. 4, all simulation parameters are kept the same as in Fig. 3. Clearly, the phase difference has been greatly reduced. For the case of the weak scattering, $g$ and $h$ are close to unity $(g=h$ $=1.01$; the agreement between the exact solution and the DWBA is nearly perfect. Because of its inherent advantage, the DWBA is able to describe the scattering by objects with complex shapes, orientations, and inhomogeneous material properties, and has been successfully applied to the zooplankton scattering. ${ }^{2-5}$

\section{PHASE-COMPENSATED DWBA}

Careful inspection of Fig. 4 reveals that for a weakly scattering object, the modulus of the scattering amplitude computed from the DWBA agrees reasonably well with the exact solution. Since Eq. (7) provides only the real part of the scattering amplitude, it indicates that the real component 

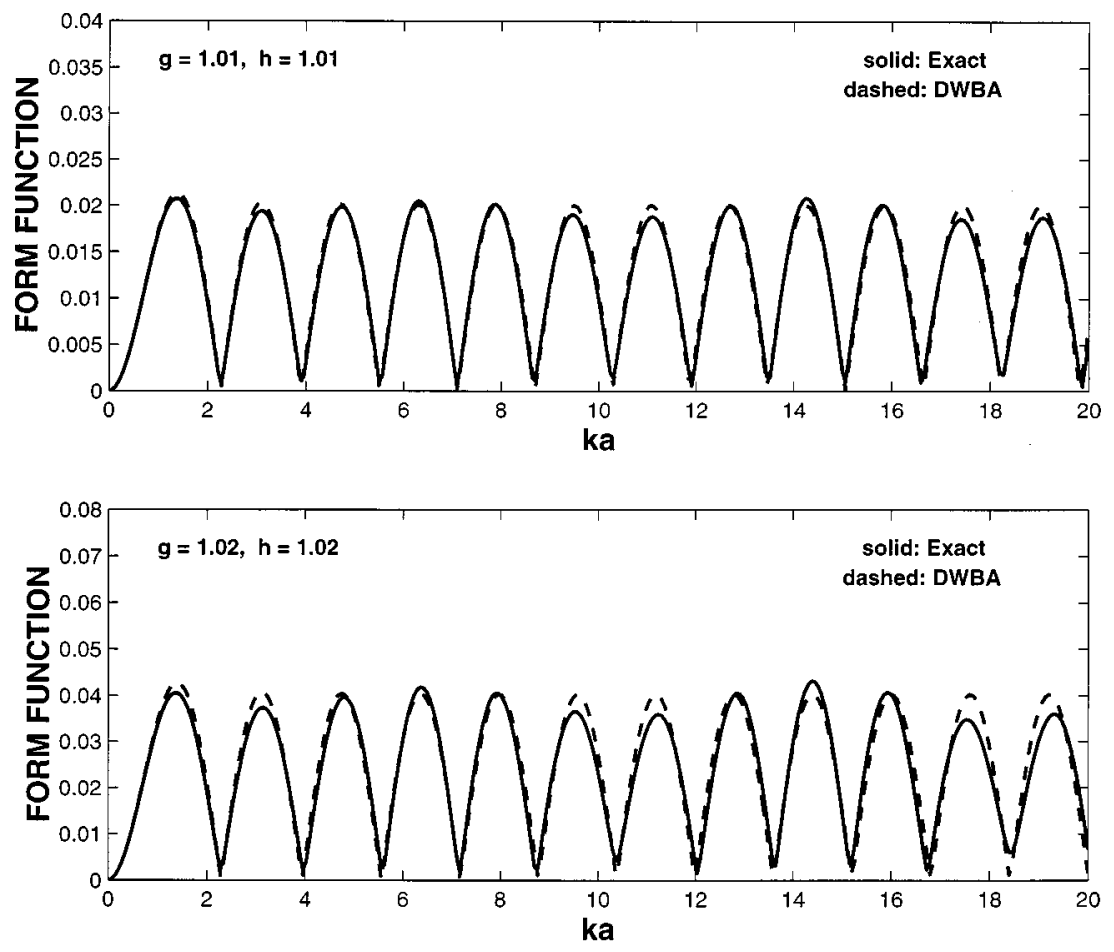

FIG. 4. Comparison of the backscattering form function $\left(\theta_{s}=180^{\circ}\right)$ of a fluid sphere between the exact solution and the distorted wave Born approximation (DWBA) with different combinations of the density and sound-speed contrasts $(g$ and $h$ ). The solid lines are computed from the exact solution (Ref. 9) and the dashed lines are computed from Eq. (7).

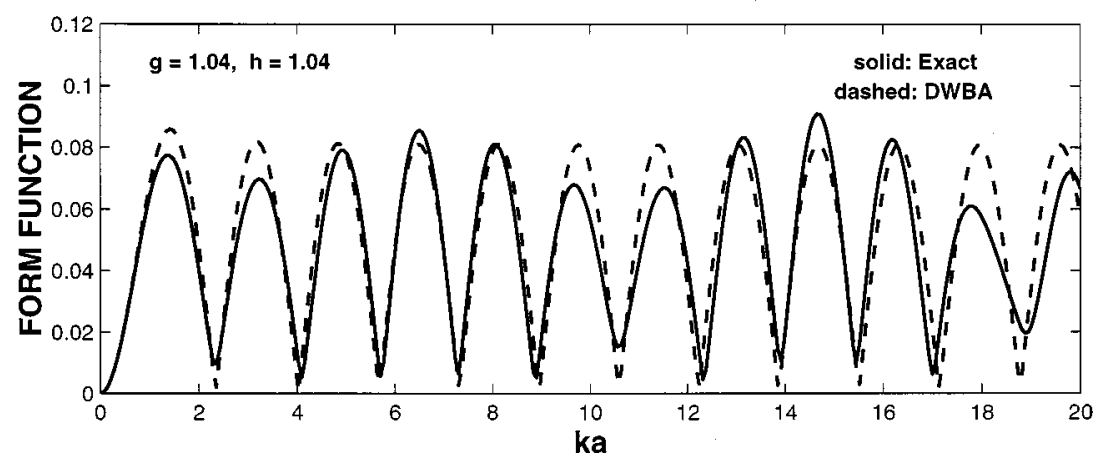

of the scattering amplitude dominates the scattering process, while the imaginary component is much smaller and is ignored in the DWBA model. For the forward scattering, $\mathbf{k}_{i}$ $=\mathbf{k}_{s}$, the integrand term $e^{i\left(\mathbf{k}_{i}-\mathbf{k}_{s}\right)}$ in both Eq. (5) and Eq. (7) is equal to 1 , and the resultant scattering amplitude $f_{\text {scat }}^{\text {DWBA }}(0)$, regardless of the shape and orientation of the object, is always a real function. For the bistatic scattering, the imaginary part is canceled out due to the symmetric gemetry of a sphere. Clearly, in its present form, the DWBA cannot predict the scattering-induced attenuation characterized by the imaginary part of the scattering amplitude used in Eq. (2). For a general 3-D scattering problem, it is not easy, at least not obvious, to find a complex scattering amplitude representation which is capable of describing both modulus and phase reasonably well.

Without losing generality, we start with the simplest 1-D problem as shown in Fig. 5: an infinite medium containing a finite homogeneous layer with a thickness of $2 H$. First, we use the DWBA method to derive a solution for this 1-D problem. Under DWBA, the internal field $P_{\text {int }}\left(z_{0}\right)$ and its Green's function $G\left(z \mid z_{0}\right)$ can be expressed as

$$
P_{\text {int }}\left(z_{0}\right)=e^{i k_{1} z_{0}}, \quad G\left(z \mid z_{0}\right)=\frac{i}{2 k} e^{ \pm i k_{1}\left|z_{0}-z\right|}
$$

where \pm signs correspond to $z_{0}>z$ and $z_{0}<z$, respectively. By substituting Eq. (8) into the original integral Eq. (3), the backscattering and forward scattering can easily be obtained by integrating Eq. (3) directly,

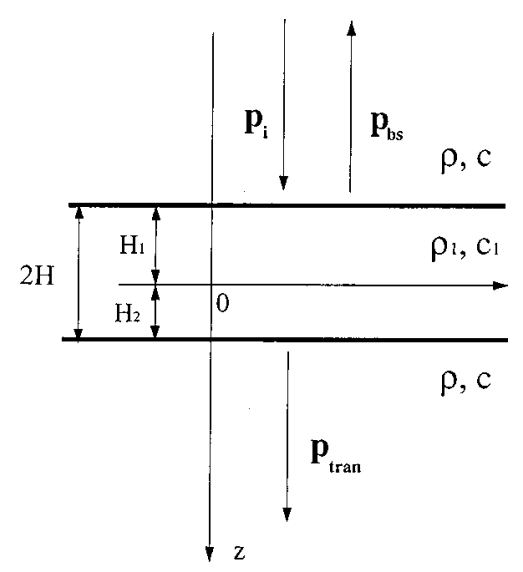

FIG. 5. Geometry of scattering by a homogeneous fluid layer. The incident wave is a plane wave. The boundaries of the layer are located at $z=-H_{1}$ and $z=H_{2}$, respectively. The thickness of the layer is $2 \mathrm{H}$. 


$$
\begin{aligned}
p_{\mathrm{bs}}^{\mathrm{DWBA}} & =-\frac{h}{4}\left(h^{2} \gamma_{k}-\gamma_{\rho}\right) e^{-i 2 k_{1} H_{1}}\left(1-e^{i 4 k_{1} H}\right) e^{-i k z} \\
& \approx \frac{1}{2}(\Delta h+\Delta g) e^{-i 2 k_{1} H_{1}}\left(1-e^{i 4 k_{1} H}\right) e^{-i k z}, \\
p_{\mathrm{fwd}}^{\mathrm{DWBA}} & =-i h k H\left(h^{2} \gamma_{k}+\gamma_{\rho}\right) e^{i k z} \approx-i \Delta h k H e^{i k z},
\end{aligned}
$$

where $h=c_{1} / c$ and $g=\rho_{1} / \rho$ are the sound-speed and density contrasts, respectively. $\Delta h=h-1$ and $\Delta g=g-1$ are the deviations of the sound-speed and density contrasts from unity.

Now, consider the exact solution. It is easy to show that the exact solutions for backscattered (reflected) and forward scattered (transmitted-incident) fields are

$$
\begin{aligned}
& p_{\mathrm{bs}}^{\mathrm{EXACT}}=R e^{-i 2 k H_{1}} \frac{\left(1-e^{i 4 k_{1} H}\right) e^{-i k z}}{1-R^{2} e^{i 4 k_{1} H}}, \\
& p_{\mathrm{fwd}}^{\mathrm{EXACT}}=T e^{i 2\left(k_{1}-k\right) H} \frac{(1-R) e^{i k z}}{1-R^{2} e^{i 4 k_{1} H}}-e^{i k z},
\end{aligned}
$$

where $R$ and $T$ are reflection and transmission coefficients defined as

$$
R=\frac{g h-1}{g h+1}, \quad T=\frac{2 g h}{g h+1} .
$$

It is not yet obvious to directly see the differences between the exact and the DWBA solutions. To relate the two results, we take the advantage of the weak scattering assumption: $g \approx 1$ and $h \approx 1$, which leads to $R \ll 1, T \approx 1$. With some straightforward mathematical manipulations, we obtain

$$
\begin{aligned}
p_{\mathrm{bs}}^{\mathrm{EXACT}} & \approx \frac{1}{2}(\Delta h+\Delta g) e^{-i 2 k H_{1}}\left(1-e^{i 4 k_{1} H}\right) e^{-k z} \\
& =e^{i \Phi_{\mathrm{bs}}} p_{\mathrm{bs}}^{\mathrm{DWBA}} \\
p_{\mathrm{fwd}}^{\mathrm{EXACT}} & \approx-i \Delta h k\left(1-i \Delta h k_{1} H\right) e^{i k z} \\
& \approx-i \Delta h k e^{-i \Delta h k_{1} H} e^{i k z}=e^{i \Phi_{\mathrm{fwd}}} p_{\mathrm{fwd}}^{\mathrm{DWB}},
\end{aligned}
$$

where the two phase terms are

$$
\Phi_{\mathrm{bs}}=-2 \Delta h k_{1} H_{1}, \quad \Phi_{\mathrm{fwd}}=-\Delta h k_{1} H .
$$

The amplitudes computed from both exact and the DWBA models are about the same, but their phases differ by factors of $-2 \Delta h k_{1} H_{1}$ and $-\Delta h k_{1} H$ for the backscattering and forward scattering, respectively. If we choose the center of the layer as the origin, i.e., $H_{1}=H$, Eqs. (15) can be formulated in a more general form,

$$
\Phi_{1 d}=-\left[1+\sin \left(\theta_{s} / 2\right)\right] \Delta h k_{1} H,
$$

where $\theta_{s}$ is the scattering angle (angle between incident and scattering directions), $\theta_{s}=0^{\circ}$ for forward scattering, and $\theta_{s}$ $=180^{\circ}$ for backscattering. The factor $\Phi_{1 d}$ is the compensated phase.

For a three-dimensional finite object, the phase compensation becomes more complicated. Let us consider the case of a sphere. Because of the finite dimension and the curved surface, the analytical solution, unlike the 1-D case, is a summation of an infinite series of partial waves (modes) involving Bessel and Legendre functions and is difficult to manipulate mathematically. However, we may utilize the result derived for the one-dimensional case. We take the following
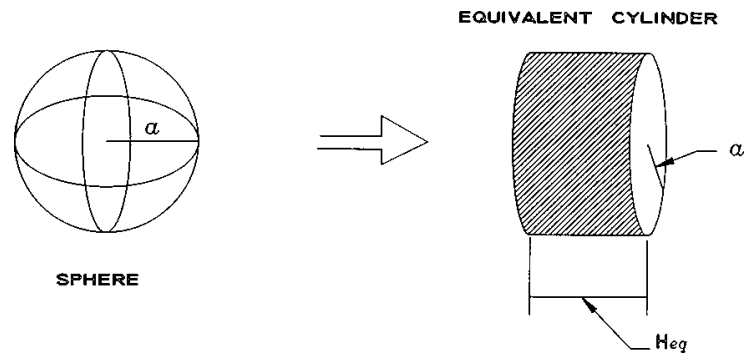

FIG. 6. Schematic of an equal volume cylinder as an approximate to a sphere, where the cylindrical radius is the same as the radius of the original sphere $a$ and the geometric cylindrical cross section in the direction of incident is $\pi a^{2}$.

heuristic approach: first, transform the 3-D problem to a quasi-1-D problem, then seek an approximate phase compensation term similar to Eq. (16). To do this, in the incident direction we squeeze the sphere from both ends into a cylinder whose cylindrical radius is $a$, while keeping the volume and the maximum geometric cross section unchanged as illustrated in Fig. 6. The backscattering and the forward scattering from such an equal volume cylinder can be approximately regarded as a quasi-1-D problem when $k a \gg 1$ (wavelength $\lambda \ll a$ ). The equivalent thickness of the cylinder is found to be $H_{\text {eq }}=2 a / 3$. If we choose the center of the sphere of radius $a$ as the origin of the coordinates, following Eq. (16), we obtain the quasi-1-D phase compensation for a sphere,

$$
\Phi_{\text {sphere }}=-\frac{2}{3}\left[1+\sin \left(\theta_{s} / 2\right)\right] \Delta h k_{1} a,
$$

where scattering angle $\theta_{s}$ is defined in Fig. 2. Substituting the phase compensation term Eq. (17) into the original DWBA solution Eq. (7), we obtain a phase-compensated DWBA representation of the scattering by a weakly scattering fluid sphere

$$
\begin{aligned}
f_{\text {scat }}^{\mathrm{PC}-\mathrm{DWBA}} & \left(\theta_{s}, k a\right) \\
= & k_{1}^{2} a^{3}\left(h^{2} \gamma_{k}+\gamma_{\rho} \cos \theta_{s}\right) \frac{j_{1}\left(2 k_{1} a \sin \left(\theta_{s} / 2\right)\right)}{2 k_{1} a \sin \left(\theta_{s} / 2\right)} \\
& \times e^{-i 2 / 3\left[1+\sin \left(\theta_{s} / 2\right)\right] \Delta h k_{1} a} .
\end{aligned}
$$

Note that the above equation is applicable not only to the forward scattering and backscattering, but also to a more general bistatic scattering problem.

To validate this approach, the above solution is compared with the exact solution. Figure 7 shows the comparison between the exact solution based on the Anderson's fluid sphere $\operatorname{model}^{9}$ (solid) and the phase-compensated DWBA solution (dashed) given by Eq. (18). It is found that both real and imaginary components of the scattering amplitude agree with the exact solution reasonably well. The sound-speed and density contrasts, $h, g$, used in the comparison are both 1.04. For zooplankton applications, $h=1.04$ and $g=1.04$ are considered to be quite reasonable. ${ }^{4,10,11}$ Because of the nature of the DWBA, it is expected that smaller $h$ and $g$ will give a better fit, while larger $h$ and $g$ will degrade the agreement.

It is obvious that the simple phase compensation Eq. (17) basically captures the phase of the scattering amplitude well. More importantly, the phase-compensated DWBA is 

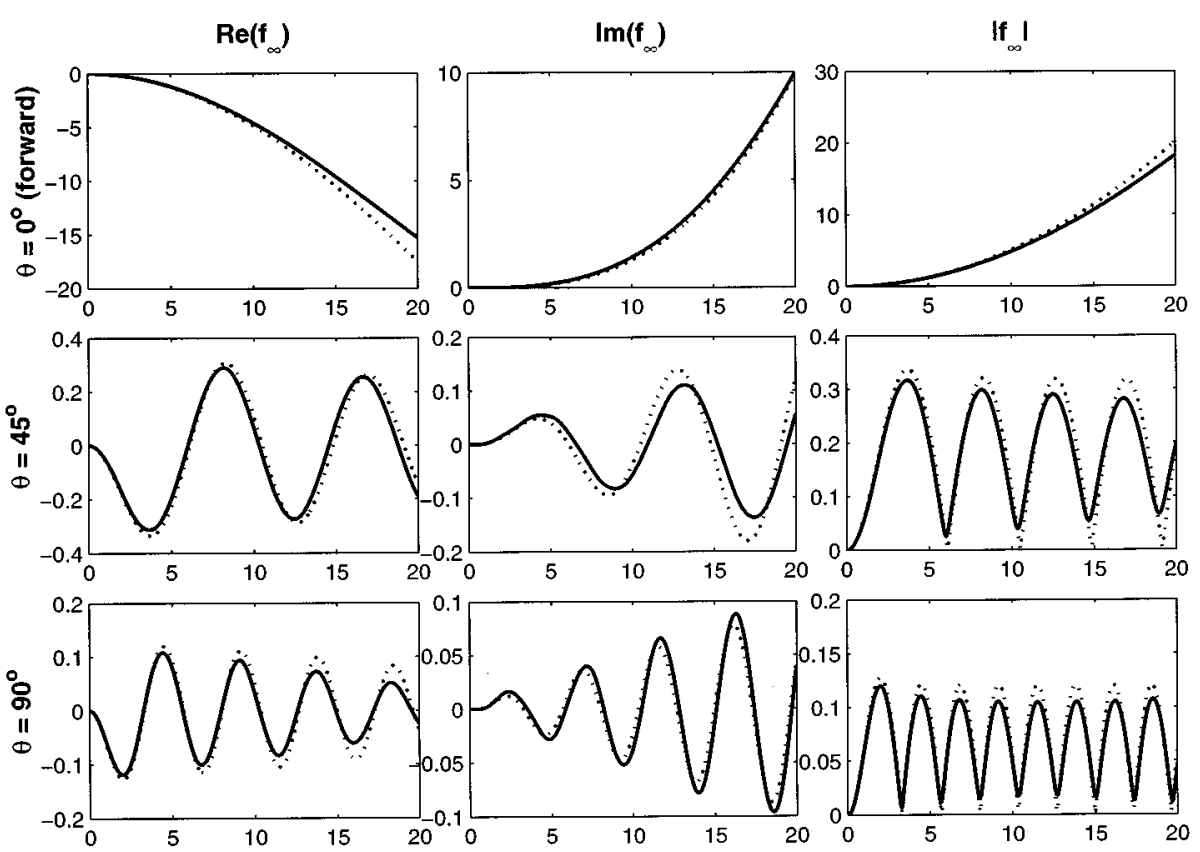

. Comparison of bistatic scattering form function of a fluid sphere between the exact solution and the phase-compensated DWBA solution. The solid lines are computed from the exact solution (Ref. 9) and the dashed lines are computed from Eq. (18). The simulation parameters are $g=h$ $=1.04 . \theta_{s}=0^{\circ}$ for forward scattering and $\theta_{s}=180^{\circ}$ for backscattering.
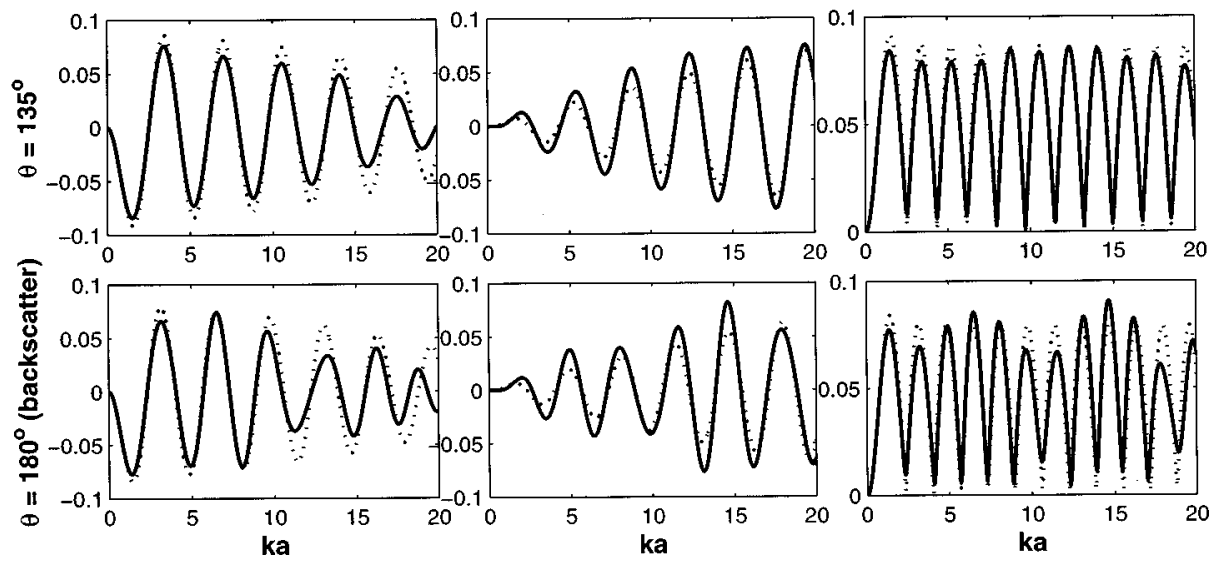

now capable of predicting the scattering-induced attenuation when used in describing propagation problems.

\section{APPLICATIONS TO ZOOPLANKTON}

Although many marine animals such as copepods, an important zooplankton class, have been successfully modeled as spheres in predicting the biomass distributions, ${ }^{12,13}$ many marine organisms have elongated shapes and have been successfully modeled as deformed cylinders for both laboratory experiments and field applications. ${ }^{2-5}$ However, these models are restricted to the backscattering case and are not able to predict any scattering-induced attenuation.

In the previous section, we have presented the phasecompensated DWBA solution for a fluid sphere. To make it more general, we model the elongated marine organisms as prolate spheroids. First, we need to find the DWBA representation of the bistatic scattering by performing a 3-D integral of Eq. (7). For a broadside incidence of a plane wave on a fluid prolate spheroid shown in Fig. 8(a), the DWBA solution is [Appendix, Eq. (A20)]

$$
\begin{gathered}
f_{\text {scat }}^{\operatorname{DWBA}}\left(\theta_{s}, k a, e\right)=k_{1}^{2} a^{3} e\left(h^{2} \gamma_{k}+\gamma_{\rho} \cos \theta_{s}\right) \\
\times \frac{j_{1}\left(k_{1} a \Phi\left(\theta_{s}, e\right)\right)}{k_{1} a \Phi\left(\theta_{s}, e\right)}, \\
\Phi\left(\theta_{s}, e\right)=\sqrt{\left(1-\cos \theta_{s}\right)^{2}+e^{2} \sin ^{2} \theta_{s}},
\end{gathered}
$$

where $e=b / a>1$ is the aspect ratio of the prolate spheroid, and $a$ and $b$ are its semiminor and semimajor axes, respectively. For $e=1$, Eq. (19) reduces to Eq. (7). Clearly, Eq. (19) has only the real part. To obtain the imaginary part of the scattering amplitude, we have to seek a phase compensation term as in the previous sphere case. One possible solution would be simply following the procedures that lead to the phase compensation term for the sphere case by squeezing the scatter in the direction of the incident wave while maintaining the volume $\left(V=4 / 3 \pi a^{2} b\right)$ and leaving the maximum geometric cross section in that direction $(S$ $=\pi a b)$ unchanged. The equivalent thickness of such a quasi-1-D problem would be $H_{\mathrm{eq}}=2 a / 3$. However, because of the complicated nature of the scattering problem, the above solution does not provide the best result. For a prolate spheroid, the curvature on the surface is not a constant; 


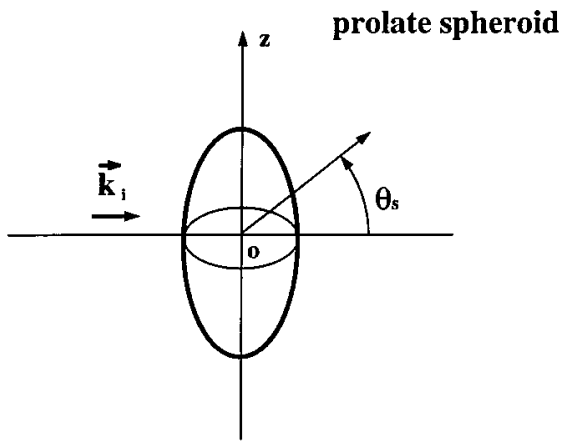

(a)

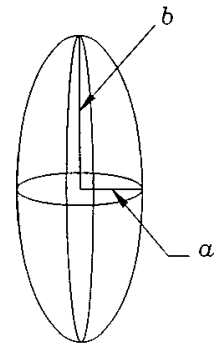

PROLATE
SPHEROID

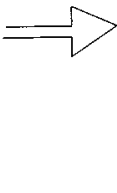

(b)

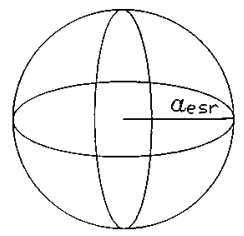

EQUIVALENT SPHERE
FIG. 8. Scattering by a prolate spheroid. (a) Geometry of bistatic scattering subject to a plane wave for a broadside incidence. $\theta_{s}=0^{\circ}$ for forward scattering and $\theta_{s}=180^{\circ}$ for backscattering. (b) Schematic of conversion from a prolate spheroid to an equivalent sphere of equal volume.

squeezing the prolate spheroid along the minor axis (direction of incidence) while keeping the maximum geometric cross section unchanged can be regarded as only using a constant radius of curvature, $a$. To overcome such a deficiency, the concept of the equivalent spherical radius commonly used in describing the scattering by irregular-shaped objects can be used, and is shown in Fig. 8(b). Since the equivalent spherical radius $a_{\mathrm{esr}}=a e^{1 / 3}$ is between $a$ and $b$, using $a_{\text {esr }}$ implies using an average radius of curvature between $a$ and $b$. The resultant equivalent thickness for this quasi-1-D problem is then found to be

$$
H_{\mathrm{eq}}=\frac{2 a_{\mathrm{esr}}}{3}=\frac{2\left(a^{2} b\right)^{1 / 3}}{3}=\frac{2 a}{3} e^{1 / 3} .
$$

As a result, the phase compensation term for a fluid prolate spheroid can be obtained by replacing $H$ in Eq. (16) with $H_{\text {eq }}$ given by Eq. (20)

$$
\Phi_{\text {spheroid }}=-\frac{2}{3}\left[1+\sin \left(\theta_{s} / 2\right)\right] \Delta h k_{1} a e^{1 / 3}=e^{1 / 3} \Phi_{\text {sphere }} .
$$

By combining Eq. (19) with Eq. (21), the complete solution for a prolate spheroid can be expressed as

$$
\begin{aligned}
f_{\mathrm{scat}}^{\mathrm{PC}-\mathrm{DWBA}} & \left(\theta_{s}, k a, e\right) \\
= & k_{1}^{2} a^{3} e\left(h^{2} \gamma_{k}+\gamma_{\rho} \cos \theta_{s}\right) \frac{j_{1}\left(k_{1} a \Phi\left(\theta_{s}, e\right)\right)}{k_{1} a \Phi\left(\theta_{s}, e\right)} \\
& \times e^{-i 2 / 3\left[1+\sin \left(\theta_{s} / 2\right)\right] \Delta h k_{1} a e^{1 / 3}} .
\end{aligned}
$$

Note that the above solution reduces to the sphere case, Eq. (18), if $e=1$. The comparison of the phase-compensated DWBA solution with the exact modal series solution for a broadside incidence is shown in Fig. 9, where the density and sound-speed contrasts are both chosen to be 1.04, and the aspect ratio $e$ is 5 . The exact solution is based on the result from Ref. 14. Apparently, the agreement is, in general, very promising given the fact that only a simple phase term is added. Both real and imaginary parts are described reasonably well by the phase-compensated representation, not only for monostatic scattering but also for bistatic scattering.

For objects with more complicated shapes such as zooplankton, the quasi-1-D approach may still work reasonably well provided that the scattering is weak. To show this, we express a general representation of the acoustic scattering by an arbitrarily shaped target as

$$
f_{\text {scat }}^{\mathrm{EXACT}}=\left|f_{\text {scat }}^{\mathrm{EXACT}}\right| e^{i \phi},
$$

where the phase $\phi$ is in general a function of the shape, orientation, and the material properties of the scatterer. For weakly scattering, the DWBA can predict the absolute value (module) of the complex scattering amplitude very well, i.e.,

$$
\left|f_{\text {scat }}^{\text {EXACT }}\right| \approx\left|f_{\text {scat }}^{\text {DWBA }}\right| \text {. }
$$

It is reasonable to assume that the dependence of $\phi$ on density contrast $(g)$ is very weak and can be ignored; the phase term can be then be expressed as the Taylor series expansion in terms of $\Delta h$, sound-speed contrast deviation from unity,

$$
e^{i \phi}=\exp \left(i \sum_{j=1}^{\infty} \alpha_{j} \Delta h^{j}\right)=\sum_{n=0}^{\infty} \frac{i^{n}}{n !}\left(\sum_{j=1}^{\infty} \alpha_{j} \Delta h^{j}\right)^{n},
$$

where $\alpha_{j}$ is the coefficient to be determined by the scattering geometry. For weakly scattering, $\alpha_{j} \Delta h^{j} \ll 1$, Eq. (25) can be approximated by the first two terms,

$$
e^{i \phi} \approx 1+i \alpha_{1} \Delta h .
$$

This is the first-order perturbation solution of the phasecompensated DWBA and is consistent with Eqs. (18) and (22) by setting $\alpha_{1}=-2 / 3[1+\sin (\theta / 2)] k_{1} a$ and $\alpha_{1}$ $=-2 / 3[1+\sin (\theta / 2)] k_{1} a e^{1 / 3}$, respectively.

In the case of the forward scattering, which is crucial in studying the scattering-induced attenuation [see Eq. (2)], it can be shown that the real part of $f_{\text {scat }}^{\mathrm{DWBA}}$ is independent of the shape and orientation of the scatterer, and depends only on its volume. By letting the scattering angle $\theta \rightarrow 0$, in both Eqs. (7) and (19), we arrive at

$$
f_{\text {scat }}^{\text {DWBA }}(0, k a)=\frac{k_{1}^{2} V}{4 \pi}\left(h^{2} \gamma_{k}+\gamma_{\rho}\right),
$$

for both sphere and prolate spheroid cases. To obtain the above equation, we have used the propertiy of the spherical Bessel function

$$
\lim _{x \rightarrow 0} \frac{j_{1}(x)}{x}=\frac{1}{3} .
$$

It is reasonable to further assume that the imaginary part of the scattering amplitude is also independent of the shape and orientation. In other words, for the forward scattering, the solution would be the same for objects with different shapes and/or orientations as long as the total volume is kept 


\section{SCATTERING BY A FLUID PROLATE SPHEROID}
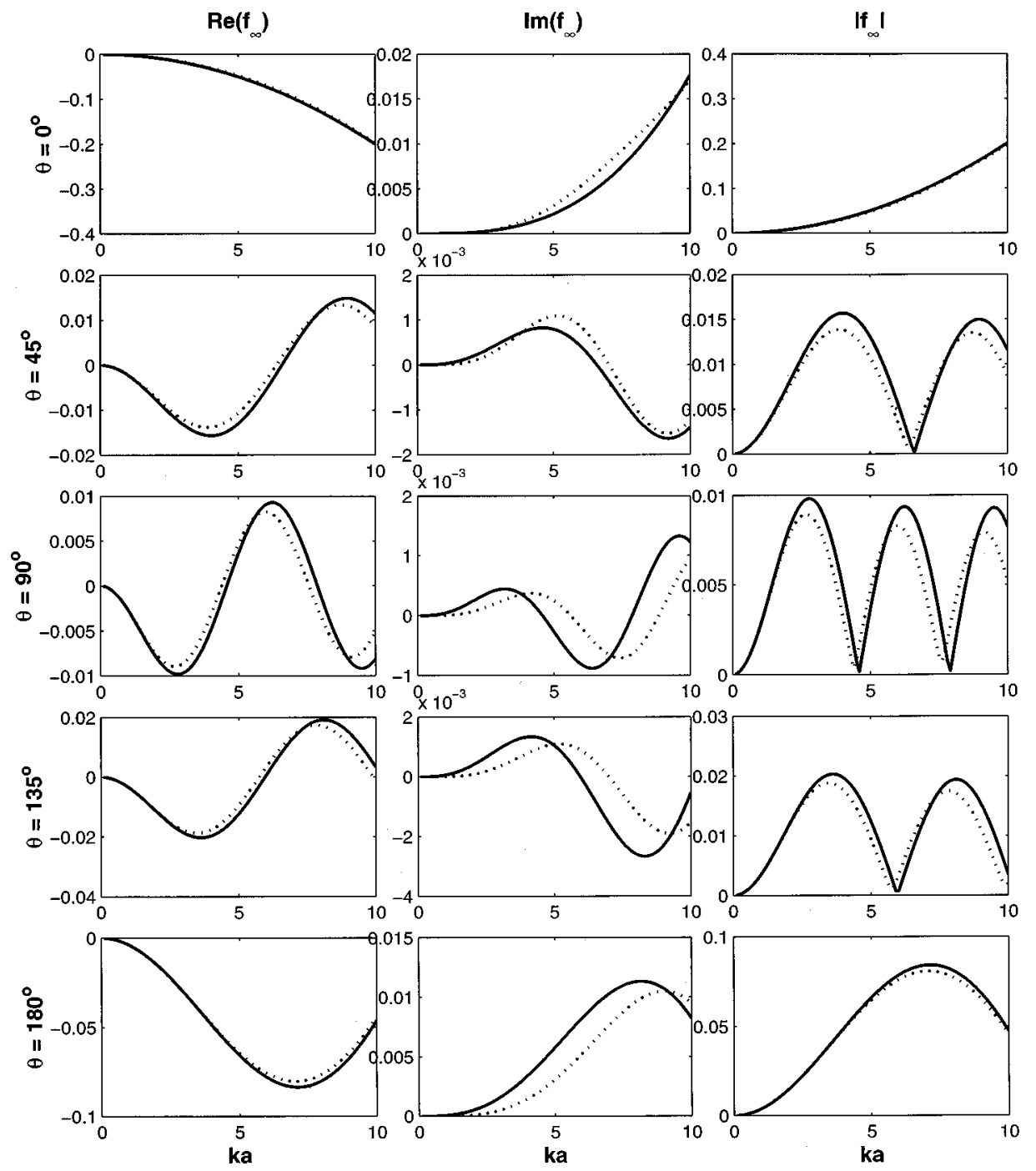

FIG. 9. Comparison of the bistatic scattering form function of a fluid spheroid between the exact solution and the phase-compensated DWBA solution. The solid lines are computed from the exact solution (Ref. 14) and the dashed lines are computed from Eq. (22). $a$ and $b$ are semiminor and semimajor axes of the prolate spheroid, respectively. The simulation parameters are $g=h=1.04$, and the aspect ratio is $e=5$.

the same. The quasi-1-D approach is basically using the concept of "equal volume."

In contrast, for a general bistatic scattering, $\alpha_{j}$ is a function of the shape and orientation of the scatterer, resulting in possible larger errors (compared to the forward scattering), but might not be unacceptable giving the condition of weakly scattering (see Fig. 9). The quantitative evaluation of such errors would involve tremendous numerical efforts and will not be discussed here.

Before applying the theory to evaluate the scatteringinduced attenuation, according to the forward-scattering theorem given by Eq. (2), we need to first understand how the complex forward-scattering amplitude depends on various parameters. For simplicity, the sphere model will be used in the following analysis. Figure 10 shows how the scattering amplitude changes as sound-speed and density contrast change. The exact solution is based on the Anderson's fluid sphere model. ${ }^{9}$ It is found that for a fixed $\Delta g$, the real part of the scattering amplitude of a fluid sphere depends on $\Delta h$ almost linearly $(\langle$ slope $\rangle \approx 0.9)$, while the imaginary part approximately depends on $\Delta h$ quadratically $(\langle$ slope $\rangle \approx 1.64)$. The slopes marked in the figure are the average slopes over a range of $\Delta h$ from 0.01 to 0.08 . To better understand such functional dependencies, we need to analysis the phasecompensated DWBA model given by Eq. (18). For forward scattering, $\theta_{s}=0$, Eq. (18) reduces to

$$
\begin{aligned}
f_{\text {scat }}^{\mathrm{PC}-\mathrm{DWBA}}(0, k a) & =\frac{k_{1}^{2} a^{3}}{3}\left(h^{2} \gamma_{k}+\gamma_{\rho}\right) e^{-i 2 / 3 \Delta h k_{1} a} \\
& \approx-\frac{k_{1}^{2} V}{4 \pi}\left(2 \Delta h+\Delta h^{2}\right)\left(1-i \frac{2}{3} \Delta h k_{1} a\right)
\end{aligned}
$$

where $V=(4 \pi / 3) a^{3}$ is the volume of the sphere. In expanding the phase term, $e^{-i 2 / 3 \Delta h k_{1} a}$, the terms of $\Delta h$ with orders higher than 1 have been ignored. As mentioned previously, the forward-scattering amplitude is independent of the shape orientation, and is proportional to the volume of the scatterer. This may be a piece of useful information that can be used in detecting marine animals by forward scatter.

To determine analytically how the real and imaginary parts of the scattering amplitude depend on $\Delta h$, we assume the relations can be approximately described by a power law, $(\Delta h)^{p}$. The power $p$ can be determined by finding the slope on a logarithm plot of the scattering amplitude versus $\Delta h$, 

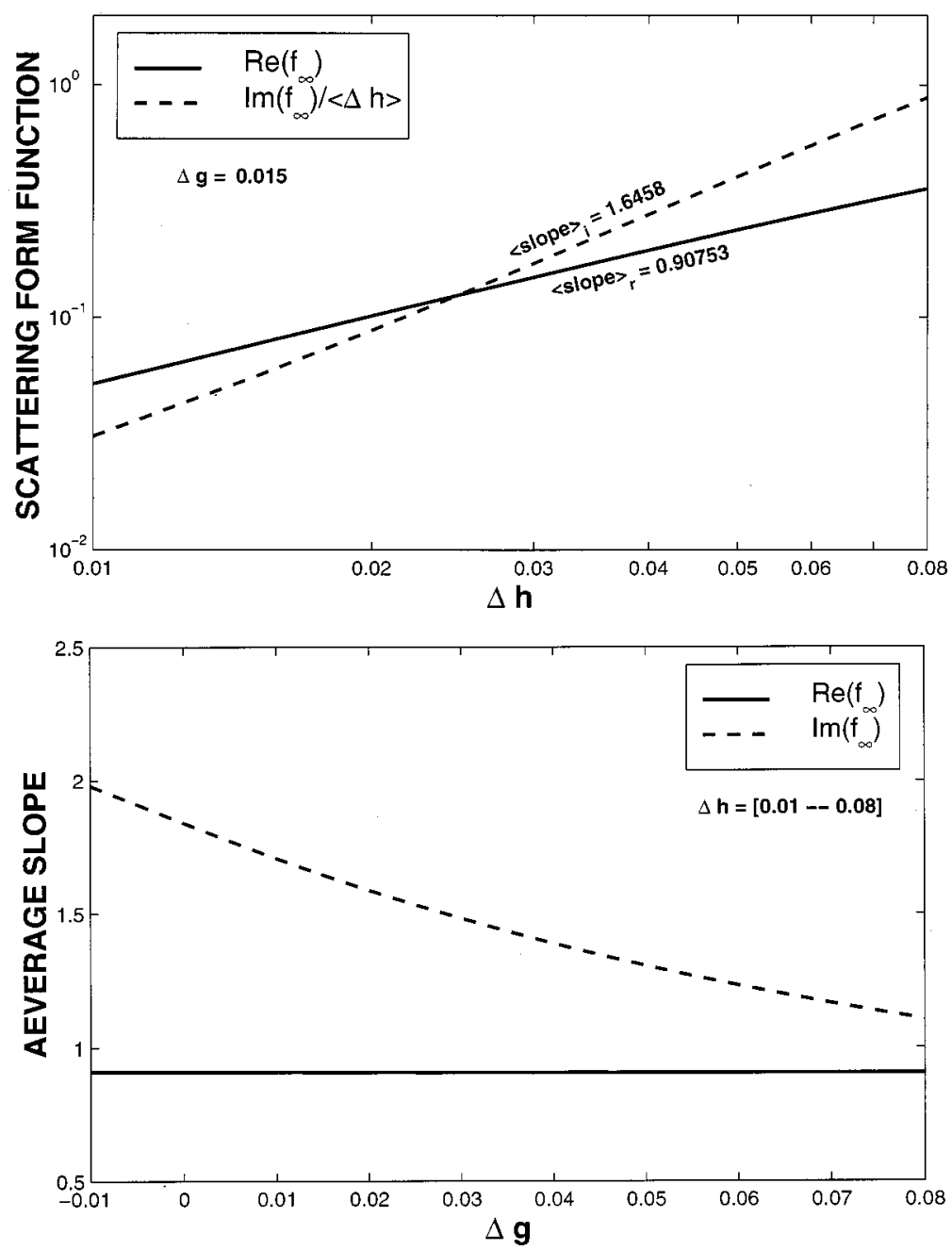

FIG. 10. Scattering form function of a fluid sphere in the forward direction $\theta=0^{\circ}$ as a function of soundspeed contrast deviation $(\Delta h=h-1)$ and density contrast deviation $(\Delta g=g-1)$. The computations are based on the exact modal series solution for a fluid sphere. The solid and dashed lines correspond to the real and the imaginary parts of the scattering form function, respectively. (a) Real and imaginary parts of the forward scattering form functions as function of $\Delta h$ with fixed $\Delta g=0.015$ and $k a=2.0$. Since $\operatorname{Im}\left(f_{\infty}\right)$ $\ll \operatorname{Re}\left(f_{\infty}\right)$, the imaginary part has been normalized by $\langle\Delta h\rangle$; (b) average slope for real and imaginary parts of forward scattering form functions versus $\Delta g$. The average over $\Delta h$ is from 0.01 to 0.08 .

$$
p=\frac{d \ln f_{\text {scat }}}{d \ln \Delta h},
$$

where $\ln$ is the natural logarithm and $f_{\text {scat }}$ could be either the real or imaginary part of the scattering amplitude. In order to apply a logarithmic operation, both real and imaginary components need to be replaced by their absolute values. From Eq. (29), by applying the chain rule of derivative, we obtain for the real part

$$
\begin{aligned}
p_{r} & =\frac{d \ln \left|\operatorname{Re}\left(f_{\text {scat }}^{\mathrm{PC}-\mathrm{DWBA}}\right)\right|}{d \ln \Delta h}=\frac{d \ln \left|\operatorname{Re}\left(f_{\mathrm{scat}}^{\mathrm{PC}-\mathrm{DWBA}}\right)\right|}{d \Delta h} \frac{d \Delta h}{d \ln \Delta h}, \\
& =\left(\frac{1}{\Delta h}+\frac{1}{2+\Delta h}-\frac{2}{1+\Delta h}\right) \Delta h=1-\frac{3}{2} \Delta h
\end{aligned}
$$

Since $\Delta h$ varies from 0.01 to 0.08 in the simulations, $\langle\Delta h\rangle=0.045$, the average slope or power, $p_{r}$, is then

$$
\left\langle p_{r}\right\rangle=1-\frac{3}{2}\langle\Delta h\rangle \approx 0.93
$$

Similarly, for the imaginary part of the scattering amplitude

$$
\begin{aligned}
p_{i} & =\frac{d \ln \left|\operatorname{Im}\left(f_{\text {scat }}^{\text {PC-DWBA }}\right)\right|}{d \ln \Delta h} \\
& =\frac{d \ln \left|\operatorname{Im}\left(f_{\text {scat }}^{\text {PC-DWBA }}\right)\right|}{d \Delta h} \frac{d \Delta h}{d \ln \Delta h}, \\
& =\left(\frac{2}{\Delta h}+\frac{1}{2+\Delta h}-\frac{3}{1+\Delta h}\right) \Delta h \\
& =2-\frac{5}{2} \Delta h,
\end{aligned}
$$

hence,

$$
\left\langle p_{i}\right\rangle=2-\frac{5}{2}\langle\Delta h\rangle \approx 1.89
$$

Comparing $\left\langle p_{r}\right\rangle$ and $\left\langle p_{i}\right\rangle$ in Eqs. (33) and (37) with those computed from the exact solutions given in Fig. 10(a), we find that the analysis based on the phase-compensated DWBA model overestimates the power $p_{r}$ and $p_{i}$, but with an error less than $4 \%$ for the real part and $16 \%$ for the imaginary part. The mismatch implies a more complicated nature of the scattering process, which depends not only on soundspeed contrast but also on density contrast. Note that, from Eqs. (33) and (37), as $\Delta h$ approaches zero, $\left\langle p_{r}\right\rangle \rightarrow 1$ (linear) and $\left\langle p_{i}\right\rangle \rightarrow 2$ (quadratic). 


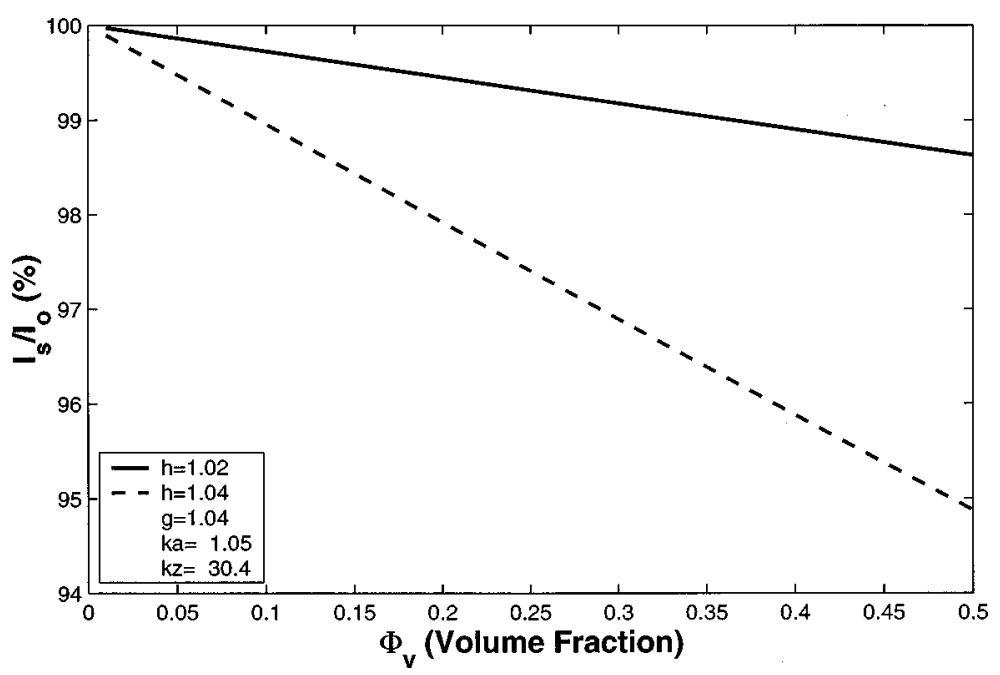

FIG. 11. Percentage of the scattering intensity $\left(I_{s} / I_{0}\right)$ observed at distance $z$ shown in Fig. 1 as a function of volume fraction of scatterers, $\Phi_{\mathrm{v}}$. The scatterers are assumed to be randomly distributed identical prolate spheroids. Two different values of $h$ have been chosen to show the dependence of attenuation on $h$. The solid line corresponds to $h=1.02$ and the dashed line corresponds to $h=1.04$, while $\Delta g$ is held constant at 0.04 . The other simulation parameters are: semiminor axis $a_{0}=0.5 \mathrm{~mm}$, aspect ratio $e_{0}=5$, acoustic frequency is $500 \mathrm{kHz}$, sound speed in water $c_{w}=1500 \mathrm{~m} / \mathrm{s}$, the distance $z=1.45 \mathrm{~cm}$. Corresponding $\mathrm{ka}$ and $\mathrm{kz}$ are 1.05 and 30.4, respectively. The PDF of the scatterers is chosen to be $P(a, e)=\delta\left(a-a_{0}\right) \delta\left(e-e_{0}\right)$.

Figure 10(b) illustrates how average slopes, $\left\langle p_{r}\right\rangle$ and $\left\langle p_{i}\right\rangle$, change as density contrast deviation, $\Delta g$, changes. As in Fig. 10(a), the computations are based on the Anderson's fluid sphere model. Obviously, the slope for the real part is essentially a constant while the slope for the imaginary part decreases from 2 to 1.1 as $\Delta g$ increases from -0.01 (a negative $\Delta g$ implies a positively buoyant scatterer) to 0.08 . This result is expected since DWBA itself is valid only for weakly scattering objects, i.e., density and sound-speed contrasts are close to unity ( $\Delta g$ and $\Delta h$ are close to zeros).

Including the imaginary part in the scattering amplitude, Eqs. (18) and (22) are able to predict the scattering-induced attenuation. Consider a unit plane wave propagation through a medium containing densely aggregated animals such as the case shown in Fig. 1. To estimate the acoustic intensity at a distance $z$, we can apply Eq. (1) and the forward-scattering theory Eq. (2) to compute the scattering-induced attenuation. Assuming the joint size and aspect ratio probability density function (PDF) of the animals is $P(a, e)$, where $a$ is the equivalent spherical radius. The average extinction cross section per unit volume due to the scattering by animals can be expressed as

$$
\left\langle\sigma_{e}\right\rangle=\frac{4 \pi n_{0}}{k} \int \operatorname{Im}\left(f_{\text {scat }}^{\mathrm{PC}-\mathrm{DWBA}}(0, k a, e)\right) P(a, e) d a d e,
$$

where $n_{0}$ is the number of scatterers in a unit volume and

$$
\int P(a, e) d a d e=1 .
$$

To evaluate Eq. (38) analytically, we choose the simplest case by setting $P(a, e)=\delta\left(a-a_{0}\right) \delta\left(e-e_{0}\right)$, where $\delta(x)$ is the Dirac delta function. The intensity at $z$ is then

$$
I_{s}(z)=I_{0} e^{-\left\langle\sigma_{e}\right\rangle z}=I_{0} e^{-4 \pi \phi_{\mathrm{V}} / k \operatorname{Im}\left(f_{\mathrm{scat}}^{\mathrm{PC}-\mathrm{DWBA}}\left(0, k a_{0}, e_{0}\right) / V\right)},
$$

where $\phi_{v}=n_{0} V$ is the volume fraction of the animal. The imaginary part of the phase-compensated DWBA solution for a prolate spheroid, $\operatorname{Im}\left[f_{\mathrm{scat}}^{\mathrm{PC}-\mathrm{DWBA}}\left(0, k a_{0}, e_{0}\right)\right]$ is given by Eq. (22). Figure 11 demonstrates the dependence of the observed intensity at location $z$ on the volume concentration $\phi_{v}$ for two different sound-speed contrasts. The intensity at $z$ decreases about $1.4 \%$ for $h=1.02(\Delta h=0.02)$, and decreases about $5.1 \%$ for $h=1.04(\Delta h=0.04)$. The decrease in intensity is nearly quadrupled for $\Delta h=0.04$ as compared to $\Delta h=0.02$, an outcome stemming from the approximate quadratic relation between the extinction cross section and $\Delta h$ as shown in Fig. 10. The implication of Fig. 11 is that once we know the volume fraction $\Phi_{v}$, by measuring the intensity attenuation, it is possible to infer the material properties of zooplankton whose influence on the acoustical scattering is significant and has been a very important issue in studying zooplankton acoustics. ${ }^{15}$

\section{CONCLUSIONS}

A phase-compensated DWBA-based model to describe the bistatic scattering by weakly scattering objects has been developed. It has been shown that the approximate phase compensation term is simple, but reasonably characterizes the general feature of the scattering for both sphere and prolate spheroid. One of the major achievements of including a phase compensation term is that the solution is able to predict the scattering-induced attenuation, which could be important when dealing with densely aggregated zooplankton.

From our studies, it is found that the imaginary part of the scattering amplitude is much smaller than the real part, approximately by a factor of $\Delta h k a$. The dependence of the scattering amplitude on the material properties of the scatterers has been investigated. The real part of the scattering amplitude is found to approximately depend on $\Delta h$ linearly, while the imaginary part is found to approximately depend on $\Delta h$ quadratically.

In reality, when the scattering-induced attenuation is noticeable, it is more convenient to measure the acoustic intensity which is directly related to the imaginary part of the forward scattering amplitude and infer the acoustic properties of the scatterers statistically.

\section{ACKNOWLEDGMENTS}

The authors would like to thank Emile Hoskinson for providing the numerical results for scattering by a prolate spheroid using the exact solutions. This work was partially 
supported by the National Science Foundation under Grant No. OCE-9730680. This is Woods Hole Oceanographic Institution Contribution No. 9819.

\section{APPENDIX}

By choosing the $z$-axis coinciding with the major axis of the prolate spheroid and defining

$$
C_{b}=h^{2} \gamma_{k}+\hat{k}_{i} \cdot \hat{k}_{s} \gamma_{\rho}
$$

which is a constant within the prolate spheroid, the first line of Eq. (7) can then be rewritten as

$$
\begin{aligned}
f_{\text {scat }}^{\mathrm{DWBA}} & =\frac{k_{1}^{2}}{4 \pi} \int_{V} C_{b} e^{i\left(\mathbf{k}_{i}-\mathbf{k}_{s}\right) \cdot r_{0}} d \mathrm{v}_{0} \\
& =\frac{k_{1}^{2} C_{b}}{4 \pi} \int_{0}^{2 \pi} d \phi_{0} \int_{0}^{r\left(z_{0}\right)} \rho_{0} d \rho_{0} \int_{-b}^{b} e^{i\left(\mathbf{k}_{i}-\mathbf{k}_{s}\right) \cdot r_{0}} d z_{0},
\end{aligned}
$$

where $b$ is the semimajor axis, and

$$
\begin{aligned}
& r_{0}=x_{0} \hat{x}+y_{0} \hat{y}+z_{0} \hat{z}, \quad \rho_{0}=\sqrt{x_{0}^{2}+y_{0}^{2}}, \\
& k_{i}-k_{s}=k_{1}\left(\hat{k}_{i}-\hat{k}_{s}\right)=k_{1}\left(\mu_{x}, \mu_{y}, \mu_{z}\right),
\end{aligned}
$$

where

$$
\begin{aligned}
& \mu_{x}=\cos \phi_{i} \sin \theta_{i}-\cos \phi_{s} \sin \theta_{s} \\
& \mu_{y}=\sin \phi_{i} \sin \theta_{i}-\sin \phi_{s} \sin \theta_{s} \\
& \mu_{z}=\cos \theta_{i}-\cos \theta_{s},
\end{aligned}
$$

with the incident and scattered unit vectors $\hat{k}_{i}$ and $\hat{k}_{s}$ defined as

$$
\begin{aligned}
& \hat{k}_{i}=\cos \phi_{i} \sin \theta_{i} \hat{x}+\sin \phi_{i} \sin \theta_{i} \hat{y}+\cos \theta_{i} \hat{z} \\
& \hat{k}_{s}=\cos \phi_{s} \sin \theta_{s} \hat{x}+\sin \phi_{s} \sin \theta_{s} \hat{y}+\cos \theta_{s} \hat{z} .
\end{aligned}
$$

Using Eqs. (A3)-(A5), we have

$$
\begin{aligned}
\left(\mathbf{k}_{i}-\mathbf{k}_{s}\right) \cdot \mathbf{r}_{0} & =k_{1}\left(\mu_{x} \rho_{0} \cos \phi_{0}+\mu_{y} \rho_{0} \sin \phi_{0}+\mu_{z} z_{0}\right) \\
& =k_{1}\left(\mu_{r} \rho_{0} \cos \left(\phi_{0}-\phi^{\prime}\right)+\mu_{z} z_{0}\right),
\end{aligned}
$$

where

$$
\begin{aligned}
& \phi_{0}=\tan ^{-1} \frac{y_{0}}{x_{0}}, \\
& \mu_{r}=\sqrt{\mu_{x}^{2}+\mu_{y}^{2}}, \\
& \phi^{\prime}=\tan ^{-1} \frac{\mu_{y}}{\mu_{x}} .
\end{aligned}
$$

Substituting Eq. (A6) into Eq. (A2), we have

$$
\begin{aligned}
f_{\text {scat }}^{\text {DWBA }} & \\
= & \frac{k_{1}^{2} C_{b}}{4 \pi} \int_{0}^{2 \pi} e^{i k_{1} \mu_{r} \rho_{0} \cos \left(\phi_{0}-\phi^{\prime}\right)} d \phi_{0} \\
& \times \int_{0}^{r\left(z_{0}\right)} \rho_{0} d \rho_{0} \int_{-b}^{b} e^{i k_{1} \mu_{z} z_{0}} d z_{0},
\end{aligned}
$$

$$
\begin{aligned}
& =\frac{k_{1}^{2} C_{b}}{2} \int_{0}^{r\left(z_{0}\right)} \rho_{0} J_{0}\left(k_{1} \mu_{r} \rho_{0}\right) d \rho_{0} \int_{-b}^{b} e^{i k_{1} \mu_{z} z_{0}} d z_{0}, \\
& =\frac{k_{1} C_{b}}{2 \mu_{r}} \int_{-b}^{b} r\left(z_{0}\right) J_{1}\left(k_{1} \mu_{r} r\left(z_{0}\right)\right) e^{i k_{1} \mu_{z} z_{0}} d z_{0},
\end{aligned}
$$

where $J_{0}(x)$ and $J_{1}(x)$ are Bessel functions of order 0 and 1 , respectively. To obtain the last equation, we have used integral properties of the Bessel function. ${ }^{16}$ For a spheroid, the $z$-dependent radius $r\left(z_{0}\right)$ can be expressed as

$$
r\left(z_{0}\right)=a \sqrt{1-\left(\frac{z_{0}}{b}\right)^{2}},
$$

where $a$ and $b$ are the semi-minor and-major axes of the prolate spheroid, respectively. Let

$$
u=\frac{z_{0}}{b}, \quad d u=\frac{d z_{0}}{b},
$$

Eq. (A10) becomes

$$
\begin{aligned}
f_{\text {scat }}^{\mathrm{DWBA}}= & \frac{k_{1} a b C_{b}}{2 \mu_{r}} \int_{-1}^{1} \sqrt{1-u^{2}} J_{1}\left(\mu_{r} k_{1} a \sqrt{1-u^{2}}\right) e^{i \mu_{z} k_{1} b u} d u \\
= & \frac{k_{1} a b C_{b}}{\mu_{r}} \int_{0}^{1} \sqrt{1-u^{2}} J_{1}\left(\mu_{r} k_{1} a \sqrt{1-u^{2}}\right) \\
& \times \cos \left(\mu_{z} k_{1} b u\right) d u .
\end{aligned}
$$

The result of the integral can be found in Ref. 17,

$$
\begin{aligned}
I & =\int_{0}^{1} \sqrt{1-u^{2}} J_{1}\left(k_{1} a \mu_{r} \sqrt{1-u^{2}}\right) \cos \left(i k_{1} \mu_{z} b u\right) d u \\
& =\mu_{r} k_{1} a \frac{j_{1}\left(k_{1} a \sqrt{\mu_{r}^{2}+e^{2} \mu_{z}^{2}}\right)}{k_{1} a \sqrt{\mu_{r}^{2}+e^{2} \mu_{z}^{2}}},
\end{aligned}
$$

where $e=b / a$ is the aspect ratio of the spheroid and $j_{1}(x)$ is the spherical Bessel function of order 1. To obtain Eq. (A14), a conversion from the cylindrical Bessel function (taken directly from Ref. 17) to the spherical function is needed by using the following relation:

$$
j_{1}(x)=\sqrt{\frac{\pi}{2 x}} J_{3 / 2}(x) .
$$

Substituting Eqs. (A14) and (A1) into Eq. (A13), the final solution can be written as

$$
f_{\text {scat }}^{\mathrm{DWBA}}=k_{1}^{2} a^{3} e\left(h^{2} \gamma_{k}+\cos \Theta \gamma_{\rho}\right) \frac{j_{1}\left(k_{1} a \sqrt{\mu_{r}^{2}+e^{2} \mu_{z}^{2}}\right)}{k_{1} a \sqrt{\mu_{r}^{2}+e^{2} \mu_{z}^{2}}},
$$

where $\mu_{r}$ and $\mu_{z}$ are defined in Eqs. (A7) and (A1), and $\Theta$ is the angle between incident $\hat{k}_{i}$ and scattered $\hat{k}_{s}$ waves

$$
\cos \Theta=\cos \theta_{i} \cos \theta_{s}+\sin \theta_{i} \sin \theta_{s} \cos \left(\phi_{i}-\phi_{s}\right) .
$$

Although Eq. (A16) is originally derived by assuming a prolate spheroid, it is also applicable to oblate spheroids by simply allowing $e<1$. 
Consider a special case of broadside incidence, in which $\theta_{i}=\pi / 2$ and $\phi_{i}=0$. If we further assume the reception is in the same plane as the incidence, we obtain

$$
\mu_{r}=1 \pm \sin \theta_{s}, \quad \mu_{z}=-\sin \theta_{s} \cos \phi_{s},
$$

where \pm signs correspond to the scattering in forward directions $(\phi=0)$ and backward directions $(\phi=\pi)$, respectively. If we define a new angle $\theta_{s}^{\prime}=\pi / 2 \mp \theta_{s}$, where $\mp$ signs correspond to the scattering in forward and backward scattering, respectively, Eq. (A18) becomes

$$
\mu_{r}=1-\cos \theta_{s}^{\prime}, \quad \mu_{z}=-\cos \theta_{s}^{\prime} .
$$

where $\theta_{s}^{\prime}$ is 0 for forward scattering and $\pi$ for backscattering, respectively. In addition, it can be deduced that $\Theta$ $=\theta_{s}^{\prime}$, implying that this new angle $\theta_{s}^{\prime}$ is just the angle between the incident and scattering directions as shown in Fig. 8 (a) $\left[\theta_{s}\right.$ in the figure is equivalent to $\theta_{s}^{\prime}$ in Eq. (A19)] ranging from 0 to $\pi$. The DWBA representation for this special case is then

$$
\begin{aligned}
f_{\text {scat }}^{\mathrm{DWBA}}= & k_{1}^{2} a^{3} e\left(h^{2} \gamma_{k}+\gamma_{\rho} \cos \theta_{s}^{\prime}\right) \\
& \times \frac{j_{1}\left(k_{1} a \sqrt{\left(1-\cos \theta_{s}^{\prime}\right)^{2}+e^{2} \sin ^{2} \theta_{s}^{\prime}}\right)}{k_{1} a \sqrt{\left(1-\cos \theta_{s}^{\prime}\right)^{2}+e^{2} \sin ^{2} \theta_{s}^{\prime}}} .
\end{aligned}
$$

Specifically, for $e=1$, Eq. (A20) reduces to the DWBA representation of the scattering amplitude by a fluid sphere,

$$
f_{\text {scat }}^{\text {DWBA }}=k_{1}^{2} a^{3}\left(h^{2} \gamma_{k}+\gamma_{\rho} \cos \theta_{s}^{\prime}\right) \frac{j_{1}\left(2 k_{1} a \sin \left(\theta_{s}^{\prime} / 2\right)\right)}{2 k_{1} a \sin \left(\theta_{s}^{\prime} / 2\right)} .
$$

For the simple Born approximation (BA), Eq. (A21) reduces to

$$
f_{\text {scat }}^{\mathrm{BA}}=k^{2} a^{3}\left(\gamma_{k}+\gamma_{\rho} \cos \theta_{s}^{\prime}\right) \frac{j_{1}\left(2 k a \sin \left(\theta_{s}^{\prime} / 2\right)\right)}{2 k a \sin \left(\theta_{s}^{\prime} / 2\right)} .
$$

${ }^{1}$ C. F. Greenlaw, "Backscattering spectra of preserved zooplankton," J. Acoust. Soc. Am. 62, 44-52 (1997).
${ }^{2}$ D. Chu, K. G. Foote, and T. K. Stanton, "Further analysis of target strength measurements of Antarctic krill at $38 \mathrm{kHz}$ and $120 \mathrm{kHz}$ : Comparison with deformed cylinder model and inference of orientation distribution,' J. Acoust. Soc. Am. 93, 2985-2988 (1993).

${ }^{3}$ T. K. Stanton, D. Chu, P. H. Wiebe, and C. S. Clay, “Average echoes from randomly oriented random-length finite cylinders: Zooplankton models," J. Acoust. Soc. Am. 94, 3463-3472 (1993).

${ }^{4}$ T. K. Stanton, D. Chu, and P. H. Wiebe, "Sound scattering by several zooplankton groups. II. Scattering Models,' J. Acoust. Soc. Am. 103, 236-253 (1998).

${ }^{5}$ P. H. Wiebe, T. K. Stanton, M. C. Benfield, D. G. Mountain, and C. H. Greene, "High-frequency acoustic volume backscattering in the Georges Bank coastal region and its interpretation using scattering models," in "Shallow water acoustics, geophysics, and oceanography," IEEE J. Ocean Eng. 22(3), 445-464 (1997).

${ }^{6}$ P. M. Morse and U. Ingard, Theoretical Acoustics (McGraw-Hill, New York, 1968), Chap. 8, pp. 407-414.

${ }^{7}$ A. Ishimaru, Wave Propagation and Scattering in Random Media (Academic, New York, 1978), Vol. I, Chap. 1, pp. 14-15, Vol. II, Chap. 14, pp. 265-268.

${ }^{8}$ M. Born and W. Wolf, Principles of Optics (Macmillan, New York, 1964).

${ }^{9}$ V. C. Anderson, “'Scattering by a fluid sphere,' J. Acoust. Soc. Am. 22, 426-431 (1950).

${ }^{10}$ K. Foote, "Speed of sound in Euphausia superba," J. Acoust. Soc. Am. 87, 1405-1408 (1990).

${ }^{11}$ K. G. Foote, T. Knutsen, A. E. Bekkevold, P. Dalpadado, and S. E. Johannessen, "Initial, collateral measurements of some properties of calanus finmarchicus," ICES C.M. 1996/L.21 Ref. B, p. 23.

${ }^{12}$ D. V. Holliday, R. E. Pieper, and G. S. Kleppel, "Determination of zooplankton size and distribution with multi-frequency acoustic technology," J. Cons., Cons. Int. Explor. Mer 46, 52-61 (1989).

${ }^{13}$ D. V. Holliday and R. E. Pieper, "Bioacoustical oceanography at high frequencies," ICES J. Mar. Sci. 52, 279-296 (1995).

${ }^{14}$ Z. Ye, E. Hoskinson, R. K. Dewey, L. Ding, and D. M. Farmer, "A method for acoustic scattering by slender bodies. I. Theory and verification," J. Acoust. Soc. Am. 102, 1964-1976 (1997).

${ }^{15} \mathrm{Z}$. Ye and S. McClatchie, "On inferring speed of sound in aquatic organisms," J. Acoust. Soc. Am. 103, 1667-1670 (1998).

${ }^{16}$ I. S. Gradshteyb and I. M. Ryzhik, Table of Integrals, Series, and Products, Corrected and Enlarged Edition (Academic, New York, 1980).

${ }^{17}$ A. Erdélyi, W. Magnus, F. Oberhettinger, and F. G. Tricomi, Tables of Integral Transformations (McGraw-Hill, New York, 1954), Vol. I, p. 57. 